\title{
Technè
}

La science au service de l'histoire de l'art et de la préservation des biens culturels

41 | 2015

Arts textiles antiques et modernes. Approche scientifique et restauration

\section{Nouvelles découvertes sur les textiles retrouvés dans le sarcophage de la reine Saddan au Tombeau des Rois (Jérusalem)}

New findings of textiles discovered in the sarcophagus of Queen Tsadan in the Tombs of the Kings (Jerusalem)

\section{Élisabeth Fontan et Christophe Moulherat}

\section{OpenEdition \\ Journals}

Édition électronique

URL : http://journals.openedition.org/techne/4227

DOI : $10.4000 /$ techne.4227

ISSN : 2534-5168

Éditeur

C2RMF

Édition imprimée

Date de publication : 25 novembre 2015

Pagination : 25-31

ISBN : 978-2-7118-6248-1

ISSN : $1254-7867$

Référence électronique

Élisabeth Fontan et Christophe Moulherat, « Nouvelles découvertes sur les textiles retrouvés dans le sarcophage de la reine Saddan au Tombeau des Rois (Jérusalem) », Technè [En ligne], 41 | 2015, mis en ligne le 08 août 2020, consulté le 11 mars 2021. URL : http://journals.openedition.org/techne/4227 ; DOI : https://doi.org/10.4000/techne.4227

\section{(c) (i) $\ominus$}

La revue Technè. La science au service de l'histoire de l'art et de la préservation des biens culturels est mise à disposition selon les termes de la Licence Creative Commons Attribution - Pas d'Utilisation Commerciale - Pas de Modification 4.0 International. 


\section{Élisabeth Fontan \\ Christophe Moulherat}

\section{Nouvelles découvertes sur les textiles retrouvés dans le sarcophage de la reine Saddan au Tombeau des Rois (Jérusalem)}

New findings of textiles discovered in the sarcophagus of Queen Tsadan in the Tombs of the Kings (Jerusalem)

Résumé. En 1863, F. de Saulcy découvrit dans le Tombeau des Rois à Jérusalem un sarcophage inviolé datant du milieu du $I^{\text {er }}$ siècle de notre ère. À l'ouverture du cercueil apparut fugitivement un squelette qui disparut aussitôt, mais les débris mêlant des restes osseux et des fils d'or à du terreau furent soigneusement recueillis au fond de la cuve et remis au Louvre. Au moment où était célébré le $150^{\circ}$ anniversaire de cette découverte, un colloque a présenté les résultats des nouvelles recherches sur le monument et a été l'occasion de lancer une étude scientifique de ces modestes vestiges de textiles.

Mots-clés. Filés or, soie, tapisserie, laine, taffetas, Tombeau des Rois, Jérusalem.
Abstract. In 1863, in the Tombs of the Kings in Jerusalem, F. de Saulcy discovered an intact sarcophagus dating from the mid-1st century AD. When the coffin was opened, a skeleton fleetingly appeared before disintegrating, but the debris, in which the remains of the bones were mixed with gold threads and earth, was meticulously collected from the bottom of the coffin and sent to the Louvre. On the 150th anniversary of this discovery, the results of the latest research into the monument were presented at a conference and a scientific study of these modest textile remains was launched.

Keywords. Gold threads, silk, tapestry, wool, taffeta, Tombs of the Kings, Jerusalem.

\section{Le Tombeau des Rois et la découverte du sarcophage de la reine Saddan}

L'exploration du Tombeau des Rois à Jérusalem au milieu du $\mathrm{XIX}^{\mathrm{e}}$ siècle a déclenché un célèbre débat qui a enflammé le monde savant. Félix de Saulcy, militaire de carrière, mais féru d'archéologie et de numismatique, étudia avec passion le monument appelé Qobour el-Molouk, qu'il identifia avec le tombeau des rois de Juda, alors que la tradition plaçait la sépulture de David et de ses descendants sur le mont Sion. Une autre hypothèse y voyait le tombeau de la reine Hélène d'Adiabène, souveraine d'un petit royaume vassal de l'Empire parthe, situé à l'est de Mossoul, qui s'était convertie au judaïsme au milieu du ${ }^{\mathrm{er}}$ siècle de notre ère. En 1863, Saulcy obtint l'autorisation d'y mener des fouilles, les toutes premières exécutées en Palestine. Il eut la chance de faire une découverte exceptionnelle dans une chambre secrète : un sarcophage inviolé, portant une double inscription au nom de la reine Saddan (fig. 1). Il relata dans ses Souvenirs d'un voyage en Terre Sainte, l'ouverture du cercueil : «... le couvercle descellé et culbuté sans recevoir une égratignure fut descendu sur le sol de la chambre. Une fois enlevé, il laissa voir un squelette bien conservé, la tête appuyée sur un coussin ou dormitoire ménagé dans la masse au fond de la cuve. De l'occiput à l'extrémité des pieds, qui avaient dû se déverser par suite de la décomposition des chairs, le cadavre mesurait $1 \mathrm{~m} 60$; il était donc de très petite taille. Toute la partie antérieure de la tête s'était effondrée et était retombée dans le fond de la boîte osseuse. Gélis, à qui j'avais recommandé de conserver précieusement la tête au moins du squelette s'il s'en trouvait un,

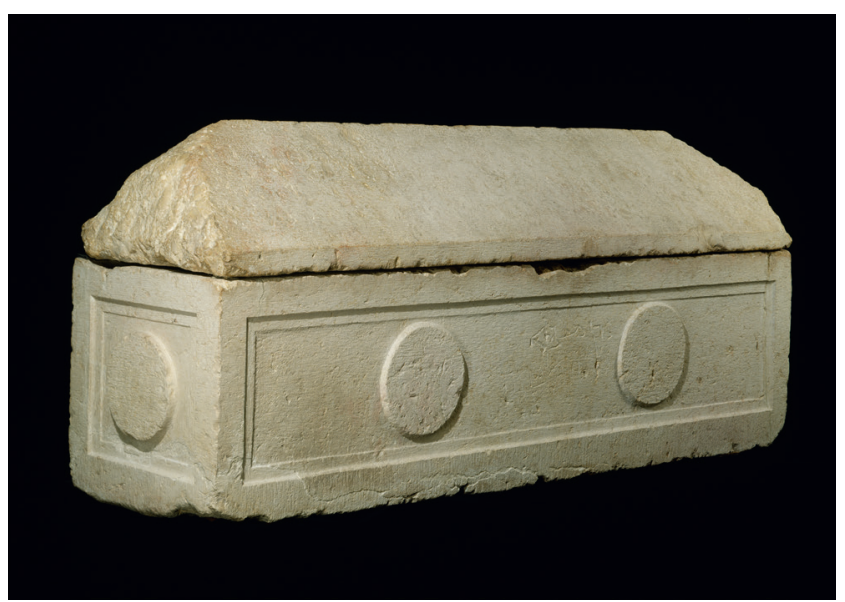

Fig. 1. Sarcophage portant une double inscription au nom de la reine Saddan. Calcaire, H. 88 ; L. 208 ; Ép. 56 cm, I Ir siècle apr. J.-C., Jérusalem, Tombeau des Rois, Don F. de Saulcy, 1864, musée du Louvre, département des Antiquités orientales, AO 5029. ๑) 2000 Musée du Louvre/Christian Larrieu.

Élisabeth Fontan, conservateur en chef honoraire, département des Antiquités orientales, musée du Louvre (elisabeth.fontan@gmail.com). Christophe Moulherat, chargé d'analyses des collections/référent textile, musée du quai Branly (christophe.moulherat@quaibranly.fr). 
comme cela paraissait probable, passa le plus délicatement qu'il put les deux mains sous le crâne, et, à l'instant même, tout s'affaissa et disparut comme par enchantement ne laissant au fond de la tombe qu'une longue tache de terreau brunâtre, mêlé d'esquilles. Ces messieurs recueillirent alors avec un soin extrême toute cette poussière, et ils purent constater que, le long du côté gauche du cadavre, il y avait des milliers de petits fils d'or tordus, d'une ténuité extrême, qui avaient dû faire partie d'une bande d'or bordant un tissu de lin assez grossier dont quelques mailles se sont conservées seules sur un fragment d'os plat ${ }^{1}$. »

Ces précieuses reliques ont été données au Louvre par Saulcy en $1870^{2}$. Elles appartenaient à un lot de petits objets provenant du Tombeau des Rois et ont été inscrites sur le livre d'entrée Napoléon III sous le numéro NIII 3540 a à e ${ }^{3}$. Des précisions furent apportées par A. Héron de Villefosse qui dressa en 1876 un catalogue des œuvres provenant de la Palestine, exposées dans la nouvelle salle judaïque qui venait d'être aménagée dans l'aile sud de la Cour Carrée ${ }^{4}$. Le n ${ }^{\circ} 22$ décrit cinq boîtes renfermant :

A. Une mâchoire inférieure trempée dans la gélatine.

B. Les rotules du genou et une troisième phalange digitale.

C. Fragments d'omoplate auxquels adhérent les maillons d'un tissu de lin bordé de fils d'or.

D. Esquilles et terreau.

E. Fils d'or tordus provenant d'une étoffe.
Le conditionnement dans des boîtes rectangulaires en carton recouvertes de papier vert, pieusement conservées jusqu'à nos jours, a été réalisé au plus tard lors de l'entrée de ces vestiges au Louvre, ou peut-être auparavant, à la demande de F. de Saulcy, car au fond de chaque boîte a été collée l'étiquette caractéristique des numéros NIII. En revanche, il est impossible de savoir si les débris conservés représentent la totalité de ce qui a été recueilli dans le sarcophage. Il est permis d'en douter. En 1912, René Dussaud publia un catalogue des collections palestiniennes et judaïques qui correspondait à la nouvelle présentation dans une salle inaugurée en 1891 sous l'Escalier Assyrien. Il reprend exactement, sous le $\mathrm{n}^{\circ} 29$, la notice d'Héron de Villefosse, en ajoutant une photographie de la mâchoire et surtout les numéros AO correspondant à l'inventaire réservé au département des Antiquités orientales, distinct de celui des Antiques, créé en 1881.

Trois de ces boîtes - C (AO5696), D (AO5692), E (AO5362) - contiennent des restes de textiles (fig. 2). Elles sont actuellement, et sans doute depuis l'entre-deux-guerres, conservées en réserve. Les fils d’or de la boîte AO5362 ont été examinés par quelques spécialistes, mais n'avaient pas jusqu'à ce jour donné lieu à une publication ${ }^{5}$.

Depuis quelques années, le ministère des Affaires étrangères a lancé un programme d'étude et de restauration du Tombeau des Rois, monument qui a été offert à la France en 1886. De nouvelles fouilles ont été menées. Dans le cadre de

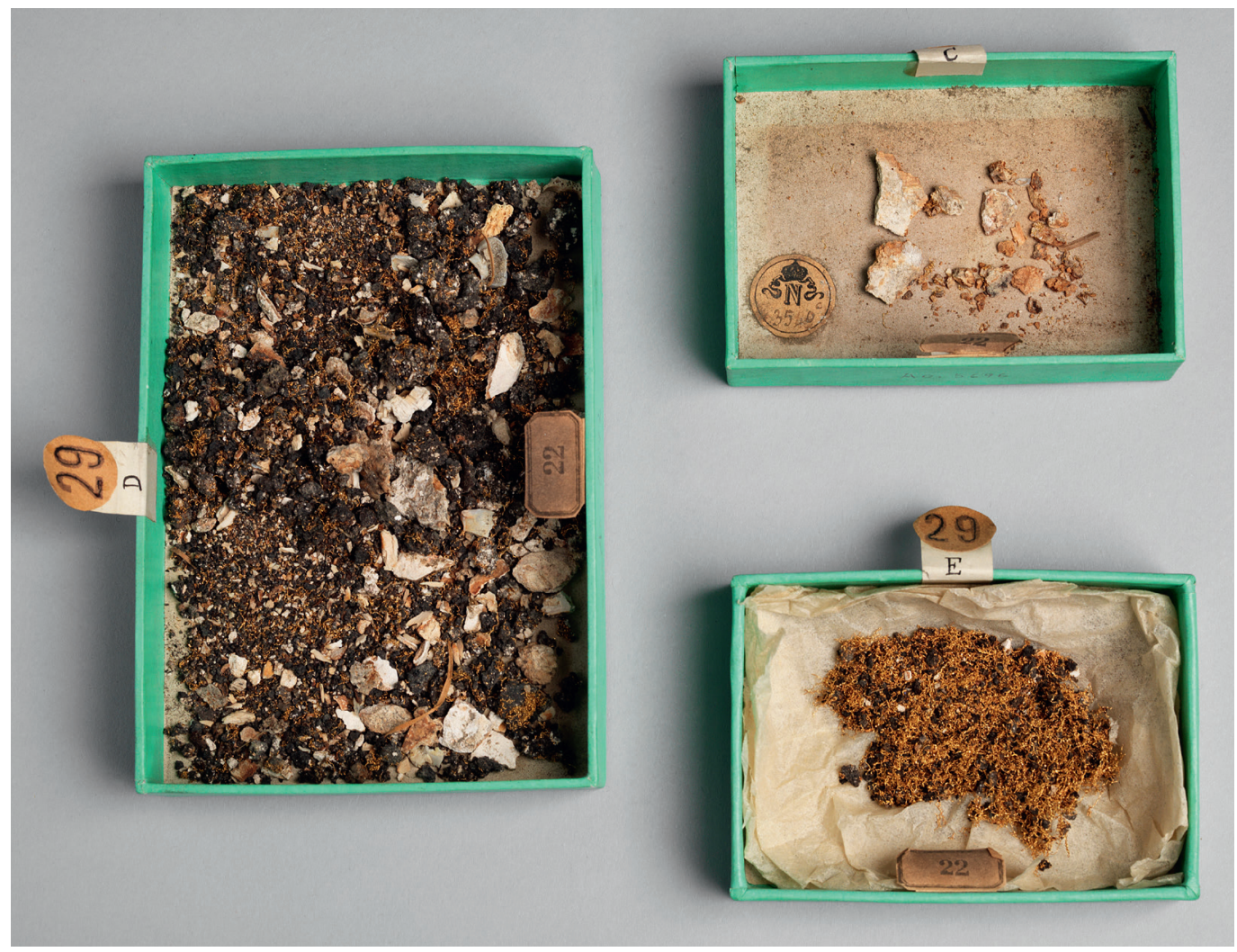

Fig. 2. Trois boîtes contenant des fils d'or, des restes osseux et autres matières recueillis dans le sarcophage de la reine Saddan, Jérusalem, Tombeau des Rois, Don F. de Saulcy, 1870, musée du Louvre, département des Antiquités orientales, AO 5692, AO 5362, AO 5696. (C) Nicolas Benoit. 
cette recherche, il a été décidé d'étudier à nouveau le matériel conservé au Louvre, notamment les restes osseux, les résidus organiques associés ${ }^{6}$ et les vestiges de textiles.

\section{L'identification des vestiges textiles}

Les observations effectuées lors de la découverte attestent que la défunte portait au moins un linceul (voir note 1). L'examen des vestiges textiles a été réalisé au musée du Louvre et au C2RMF avec le concours de Dominique Robcis, complété par des analyses effectuées au musée du quai Branly. La taille extrêmement réduite des fragments, inférieure à $5 \mathrm{~mm}$, a nécessité l'utilisation d'un équipement spécifique. Dans un premier temps, nous avons observé les fragments au moyen d'une caméra numérique 3D de marque Hirox. Puis quelques échantillons sélectionnés en fonction des premiers résultats obtenus ont été analysés au microscope électronique à balayage. Enfin, un échantillon a été inclus dans un bloc de résine afin de permettre son observation sous microscope optique.

Le résultat de ces travaux a révélé la présence de deux tissus, distincts mais étroitement associés :

- le tissu 1 est une toile qui possède un décor réalisé en tapisserie. Ce décor est en partie exécuté avec des filés or de deux diamètres différents ;

- le tissu 2 est un taffetas de soie.

Ces deux tissus ont été retrouvés l'un sous l'autre sans que l'on puisse savoir lequel était en contact direct avec le corps (fig. 3). Ils se sont conservés sous une forme minéralisée par l'action de la décomposition du corps et sont mêlés à un amalgame constitué de phosphore et de calcium qui a permis leur préservation.

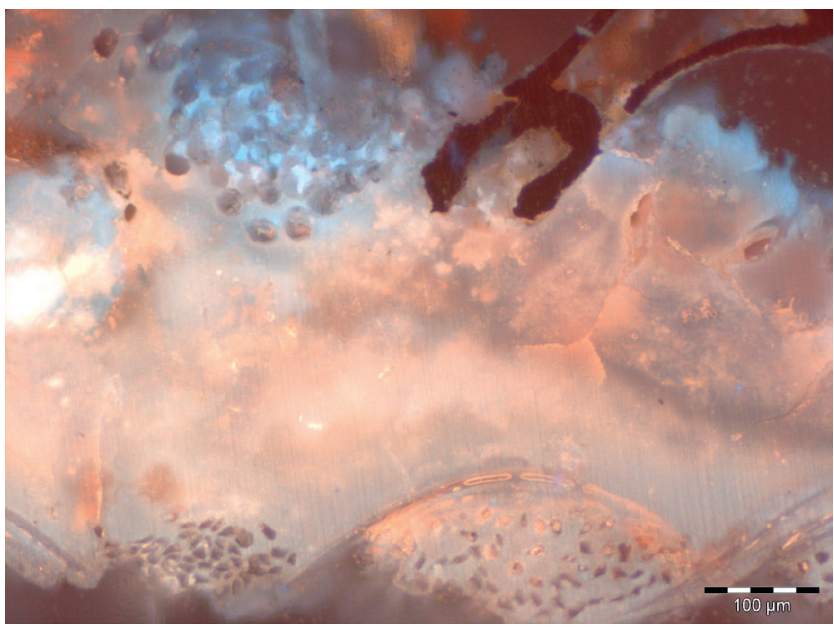

Fig. 3. Coupe qui atteste que les deux tissus étaient bien disposés l'un sur l'autre. () Musée du quai Branly/Christophe Moulherat.

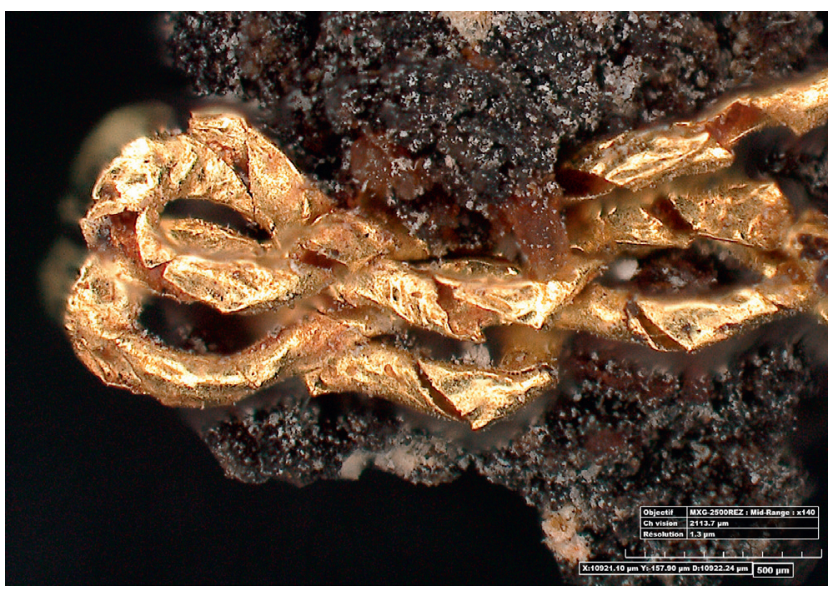

Fig. 4. Tissu 1 : boucles formées par les filés or dans le sens trame et un fil de chaîne disposé verticalement. (C) C2RMF/Dominique Robcis

\section{Description du tissu 1 (fig. 4)}

Il s'agit d'une toile dont les fils de chaîne d'un diamètre moyen de $0,20 \mathrm{~mm}$ sont en laine.

Ils sont simples, de torsion $\mathrm{Z}$ et de couleur pourprée.

La trame quant à elle se compose de deux types de fils :

- la trame de fond en laine dont la couleur n'est pas discernable ;

- la trame décorative constituée de deux types de filés or (fig. 5) qui se distinguent par leur diamètre : le diamètre du fil le plus étroit est estimé à $120 \mu \mathrm{m}$ et le second est de $130 \mu \mathrm{m}$.

Le filé or est un fil composé d'une lame de métal enroulée $\mathrm{S}$ ou $\mathrm{Z}$ autour d'une âme organique qui peut être un fil de soie, de lin ou de coton.

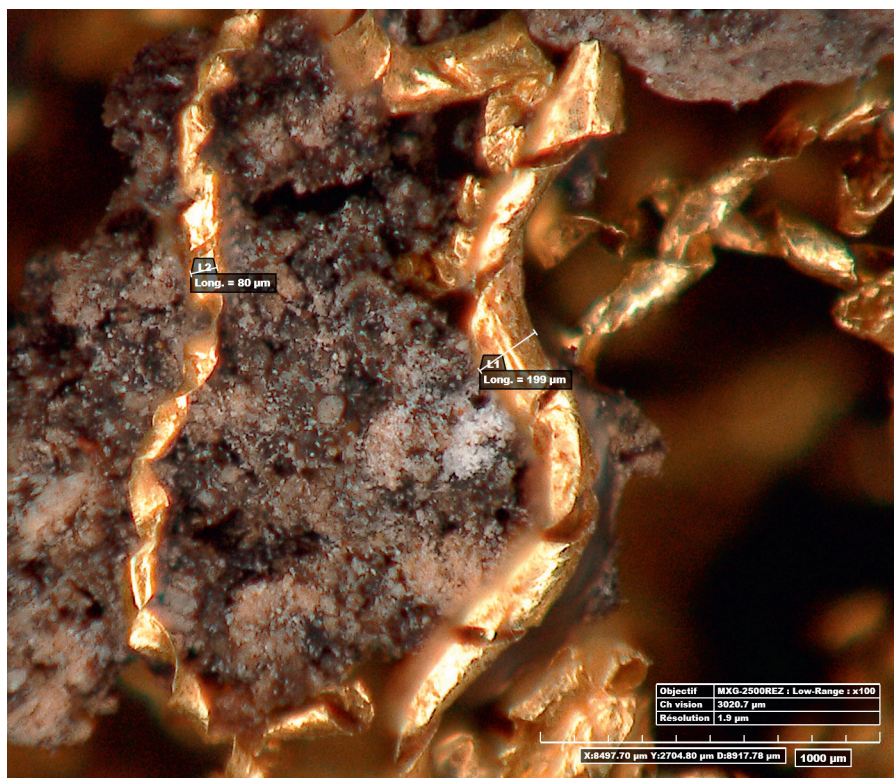

Fig. 5. Tissu 1: deux types de filés or utilisés pour le décor du tissu. (c) C2RMF/Dominique Robcis (photo réalisée sous Hirox). 
Dans l'exemple du Tombeau des Rois, il s'agit de filés de torsion $\mathrm{Z}$ autour d'une âme de torsion $\mathrm{Z}$ dont la nature n'est pas encore identifiée. Cette dernière a un diamètre de $0,08 \mathrm{~mm}$ (fig. 6).

Une série d'analyses réalisées par Maria Filomena Guerra a montré que les lames sont fabriquées en alliage d'or et que la composition de cet alliage varie selon le diamètre des lames ${ }^{7}$.

L'examen des fibres confirme la présence de laine à la fois dans le sens chaîne et dans le sens trame, et non de lin comme le notait Saulcy. Ce dernier évoque le terme de linceul de lin par référence aux coutumes de Judée, mais il est très probable qu'il s'agissait plutôt d'un vêtement rehaussé d'un décor de filés or. Ce décor a été obtenu via la technique de la tapisserie ${ }^{8}$, qui se caractérise par une trame, très fortement tassée, qui dissimule entièrement la chaîne. La taille des fragments conservés n'est pas suffisante pour apprécier la richesse du décor initial.

S'agissant des filés métalliques, il n'existe aucun témoignage de la présence d'une âme organique à l'intérieur de la spirale formée par la lame avant le $\mathrm{I}^{\mathrm{er}}$ siècle de notre ère. Deux hypothèses s'offrent à nous :

- les âmes organiques ont disparu du fait des conditions de conservation ;

- les filés métalliques ne possédaient pas d’âme organique.
La plupart des spécialistes, dont Marta Jaro notamment, excluent cette dernière hypothèse.

Ce qu'on observe, c'est l'apparition en Grèce de filés métalliques sans âme organique avant le $\mathrm{III}^{\mathrm{e}}$ siècle avant J.-C., comme l'atteste la découverte d'une urne métallique à Koropi, dans l'Attique, datée du V $\mathrm{v}^{\mathrm{e}}$ siècle avant J.-C. Les filés semblent avoir été réalisés à partir d'argent doré et utilisés pour broder des lions sur une toile de lin $^{9}$. La tombe de Derveni, non loin de Salonique, a aussi livré les restes de filés, en or cette fois, dans un contexte de la fin du IV ${ }^{\mathrm{e}}$ siècle avant notre ère ${ }^{10}$. Plus récemment, au $\mathrm{III}^{\mathrm{e}}$ siècle avant notre ère, des vestiges de filés or ont également été découverts dans une tombe d'enfant à Arta en Épire ${ }^{11}$. Tous ces exemples sans âmes organiques ne sont pas isolés.

Il faut alors attendre le $\mathrm{I}^{\mathrm{er}}$ siècle de notre ère pour observer les premiers exemples conservés de filés or avec une âme organique. Le premier provient d'un sarcophage en marbre trouvé dans le cimetière du Céramique, à l'intérieur duquel des restes de ces filés or mêlés à des vestiges de tissus pourprés ont été conservés ${ }^{12}$. Un second exemple est fourni par une coupe en argent, datée également du I ${ }^{\mathrm{er}}$ siècle ap. J.-C., conservée au Musée national d'Athènes ${ }^{14}$ sous laquelle adhèrent des filés or répartis sur une surface de $10 \mathrm{~cm}^{2}$. En Espagne également, on observe d'autres exemples conservés ${ }^{13}$ de l'utilisation de filés or avec une âme.

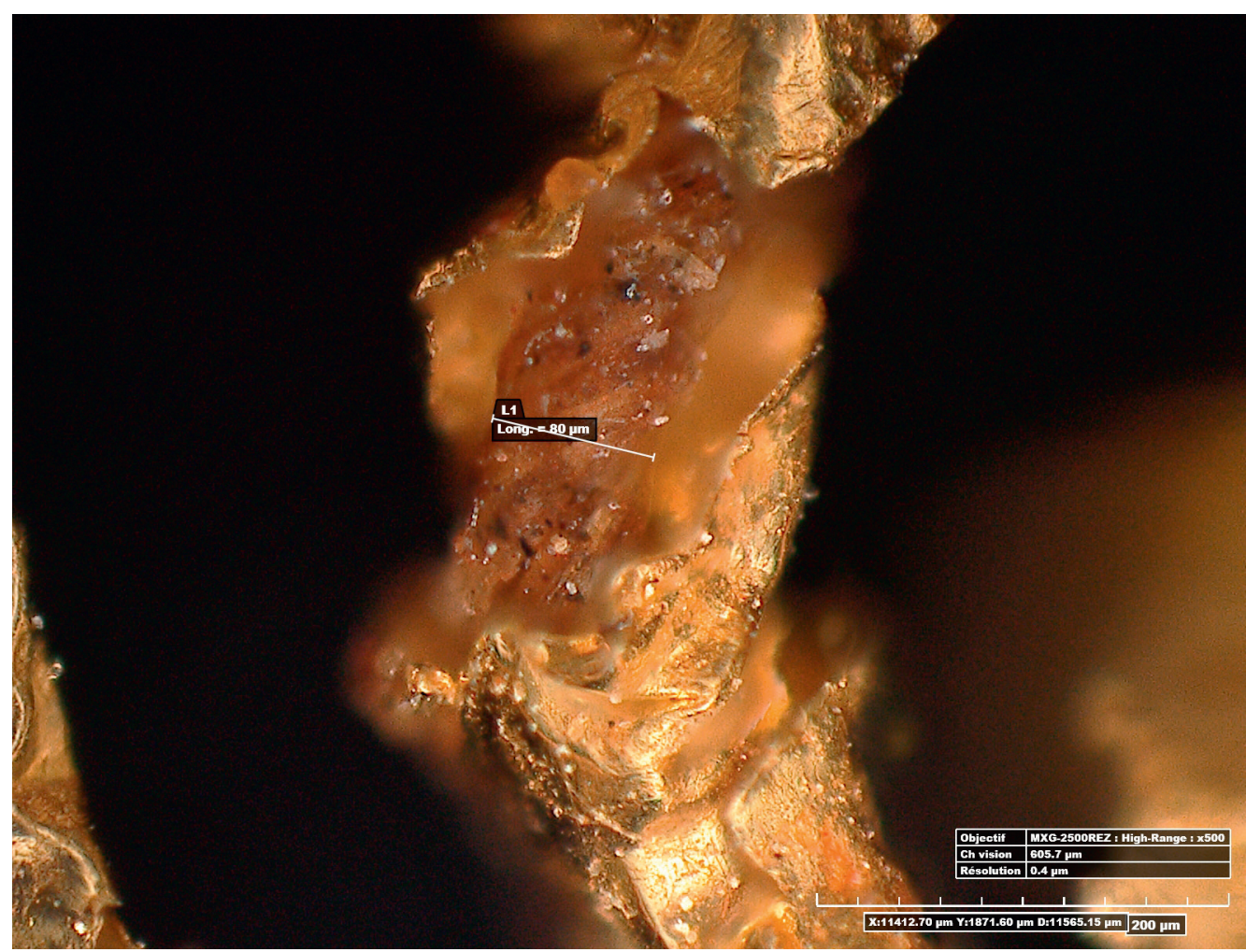

Fig. 6. Tissu 1 : fil de torsion $\mathrm{Z}$, d'un diamètre de $80 \mu \mathrm{m}$, soit $0,08 \mathrm{~mm}$. (C) C2RMF/

Dominique Robcis

(photo réalisée sous Hirox). 


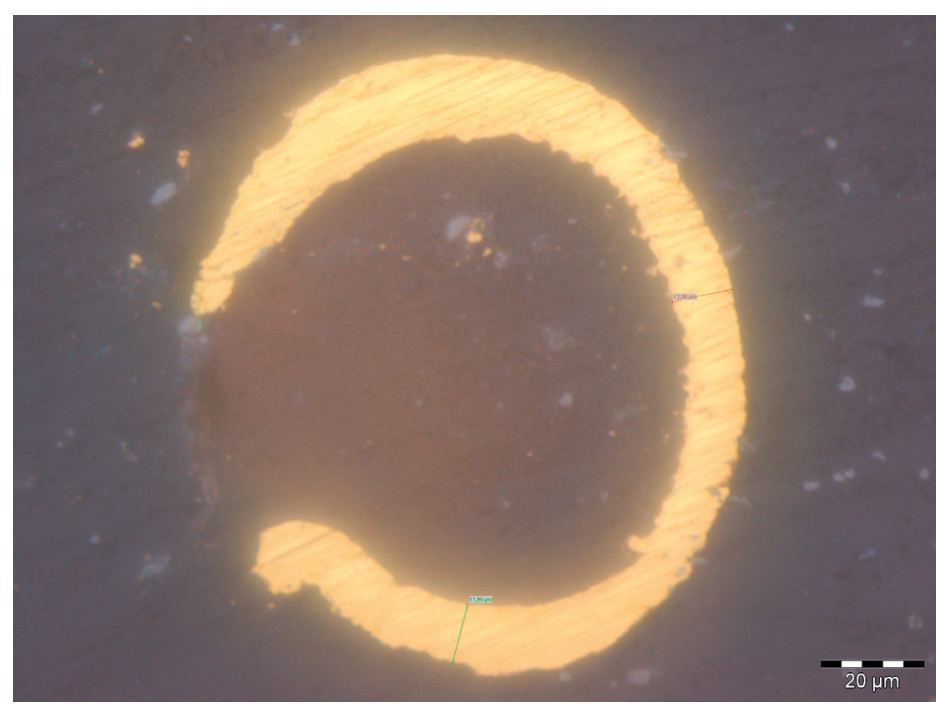

Fig. 7. Coupe avec mesure de diamètre d'un des fils d'or du tissu 1. (C) Musée du quai Branly/Christophe Moulherat.

Mais c'est à partir des $\mathrm{III}^{\mathrm{e}}$ et IV $\mathrm{IV}^{\mathrm{e}}$ siècles qu'apparaissent les témoignages les plus nombreux et les plus spectaculaires. J.-P. Wild en a dressé un premier inventaire ${ }^{15}$; mais l'essentiel du corpus se concentre sur la Rhénanie. Cet inventaire a été tout récemment complété par $\mathrm{M}$. Gleba ${ }^{16}$ qui a intégré le matériel retrouvé en Ukraine notamment.

Sur les méthodes de fabrication de ces filés métalliques, nous n'avons pas d'information directe, mais plusieurs indices situent l'origine de cette technique au Proche-Orient et plus précisément en Syrie. En effet, avec la conquête romaine apparaissent au Levant des tissus de très haute qualité dont la trame contient des fils d'or ; ces tissus servent alors à confectionner des tentures ou des vêtements de grand luxe. Selon Pline l'Ancien, cet artisanat prit naissance dans le royaume de Pergame, tout comme l'invention du tissage avec des fils d'or $^{17}$ : " aurum intexere in aedem Asia invenit Attalus rex, unde nomen Attalicis » [c'est dans cette même Asie que le roi inventa l'art de tisser avec de l'or], et « Attalici vero jam pridem intexitur, invento regum Asiae » [quant aux étoffes attaliques, il y a longtemps qu'on y tisse des fils d'or, c'est une invention des rois de l'Asie].

L'exemple du Tombeau des Rois permet d'observer très nettement la présence de véritables filés or, et on distingue parfaitement l'âme autour de laquelle s'enroule la lame métallique. Il s'agit ici d'un témoignage supplémentaire de l'apparition, dès le $\mathrm{I}^{\mathrm{er}}$ siècle de notre ère, de filés d'or avec âme organique.

Nous ne pouvons pas exclure que les premiers filés d'or observés aient pu ne pas être dotés d'une âme organique. Pour cela, il fallait que les bandes d'or aient une épaisseur suffisante pour résister à la traction des fils de chaîne dans le métier, comme le montre l'épaisseur des filés d'or des III ${ }^{\mathrm{e}}$ et $\mathrm{IV}^{\mathrm{e}}$ siècles avant notre ère. Or, dans l'exemple du Tombeau des Rois, les lames d'or ont une épaisseur d'environ $12 \mu \mathrm{m}$ (fig. 7) : elles sont deux à trois fois plus minces que celles utilisées aux périodes précédentes. Ce fait se confirmera avec la découverte des filés or d'une finesse extrême (inférieure à $10 \mu \mathrm{m}$ ) notamment à Naintré ${ }^{18}$ dans la Vienne et à Salonique dans des contextes plus tardifs (IV ${ }^{\mathrm{e}}$ siècle) ${ }^{19}$.

Après ces premières considérations sur les filés or eux-mêmes, il convient de présenter une autre particularité de ce tissu 1 : l'utilisation de ces filés selon la technique de la tapisserie. En effet, les observations sur quelques fragments ont révélé des exemples très clairs de relais, c'est-à-dire des allers-retours décrits par la trame en fonction du motif à exécuter. Mais la taille des fragments conservés n'est pas suffisante pour apprécier la richesse du décor initial.

On observe dès cette époque, et plus encore à partir des $\mathrm{III}^{\mathrm{e}}$ et IV ${ }^{\mathrm{e}}$ siècles, la présence d'étoffes à bandes à tapisserie à filé or d'un grand raffinement, non seulement parce qu'elles allient des matières de luxe - or, soie et parfois de la pourpre -, mais aussi parce qu'elles démontrent la maîtrise de savoir-faire complexes de la part des tisserands.

\section{Description du tissu 2 (fig. 8)}

Il s'agit d'un taffetas qui consiste en une armure de tissage la plus simple : l'entrecroisement rectiligne des fils de chaîne (dans la longueur du tissu) avec les fils de trame (dans la largeur), dans lequel les fils de chaîne impairs et pairs sont pris alternativement dessus et dessous, à chaque coup de

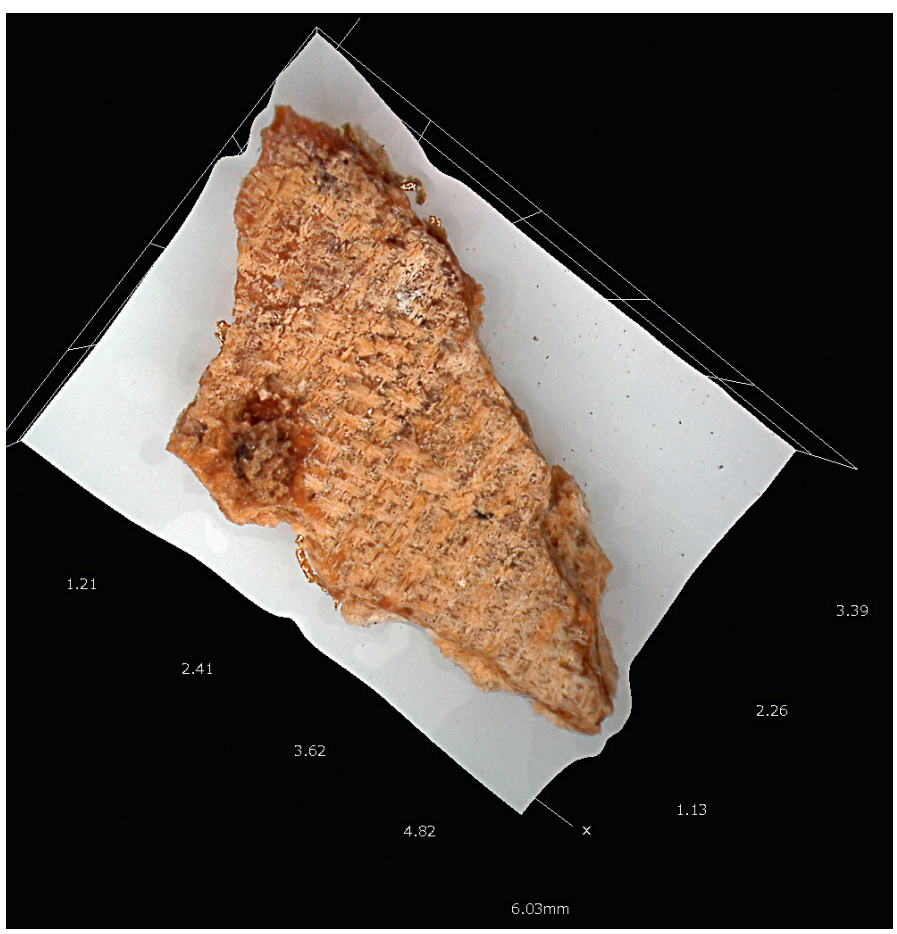

Fig. 8. Tissu 2 : vue générale d'un des fragments du taffetas. (c) C2RMF/Dominique Robcis (photo réalisée sous Hirox). 
trame. Le terme taffetas est employé pour un tissu en fibres continues, comme la soie, tandis que le terme de toile, qui correspond à la même armure, est attribué à un tissu en fibres discontinues (coton, lin, chanvre, laine...).

La chaîne et la trame n'ont pas de torsion appréciable. Ici, la chaîne est constituée de 90 fils/cm, la trame de 32 fils $/ \mathrm{cm}$. Les fils utilisés sont en soie de Bombyx Mori; près de 25 cocons ont été utilisés pour réaliser chaque fil (fig. 9).

Ce tissu peut être comparé aux taffetas de la dynastie des Han identifiés dans les sépultures Xiongnu découvertes en Mongolie et plus précisément à Noin Ula et Egin Gol. Ces sépultures datent du tout début du I ${ }^{\text {er }}$ siècle de notre ère ${ }^{20}$.

Le tissu identifié au Tombeau des Rois est l'un des plus anciens témoignages de tissu de soie découvert au Procheère pour constater la présence d'autres tissus de soie, notamment en Syrie ${ }^{21}$.

Les caractéristiques de la soie rappellent donc les productions chinoises mais en diffèrent légèrement par le nombre de cocons utilisés pour leur réalisation. En effet, les exemplaires trouvés en Mongolie montrent des fils de soie constitués d'un nombre de cocons allant jusqu'à 40.

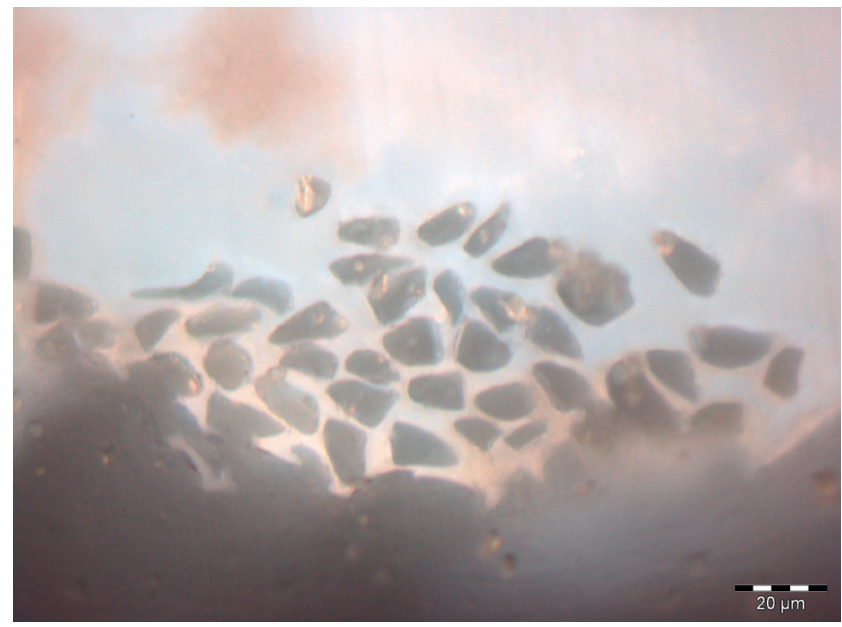

Fig. 9. Coupe du tissu 2 : section des fibres de la soie Bombyx Mori. (c) Musée du quai Branly/Christophe Moulherat.

En revanche, on retrouve une densité de fils quasi identique, autour de 90 en moyenne. De plus, dans les deux cas, les fils ont tous été décreusés après tissage, c'est-à-dire qu'ils ont subi un traitement consistant à retirer plus ou moins complètement le grès ou séricine, la matière qui maintient ensemble les deux fibroïnes de la bave.

L'originalité de cette découverte réside dans la cohabitation de deux tissus qui témoignent l'un et l'autre de deux traditions de production textile différentes. D'une part, la technique du filé or et son emploi en tapisserie, connue dans le monde occidental et en méditerranée orientale, n'est pas attestée dans la Chine des Han. D'autre part, le taffetas est une production typiquement chinoise, qui s'est diffusée à travers l'Asie centrale et par l'intermédiaire des Parthes jusqu'au Levant à partir du $\mathrm{I}^{\mathrm{er}}$ siècle de notre ère.

La présence de ce tissu d'origine chinoise pourrait être un argument supplémentaire pour identifier la femme ensevelie dans le sarcophage dit de la reine Saddan comme étant cette souveraine d'un royaume vassal de l'Empire parthe, Hélène d'Adiabène. 
Notes

1. Saulcy, 1867 , p. 244-245.

2. Archives des Musées nationaux, Procès-verbaux du conservatoire, 1BB19, séance du 9 mai 1870 : «M. de Saulcy qui a déjà si souvent enrichi les collections du Louvre vient de nouveau de donner des objets trouvés par lui dans le Tombeau des Rois à Jérusalem. »

3. Sur le registre 6DD8, l'intitulé n'est pas rempli.

4. Héron de Villefosse, 1876, p. 26-27, $\mathrm{n}^{\circ} 22 \mathrm{C}, \mathrm{D}, \mathrm{E}$.

5. Notamment Mireille Bellis, Sophie Desrosiers et Hero Granger-Taylor.

6. Étude inédite du Dr Philippe Charlier en juillet 2013. Les restes osseux avaient fait l'objet au moment de la découverte d'un examen par le Dr PrunerBey, voir Saulcy, 1865, II, p. 309 et 312 et étude de Juliette Langlois, assistante ingénieur, département Recherche, C2RMF, du 23 septembre 2014, intitulée "Résidus trouvés dans un sarcophage », compte-rendu d'étude C2RMF n 29289.

Les résultats complets de ces travaux seront présentés dans une future publication de l'ensemble des nouvelles recherches sur le Tombeau des Rois.

7. Rapport inédit de Maria Filomena Guerra, directrice de recherche au CNRS. Les résultats complets seront présentés dans la publication définitive.

8. Définition du CIETA : Genre de tissu à décor généralement polychrome, dont les trames limitent leur action aux dimensions des motifs de dessin qu'elles produisent, en couvrant totalement les fils de chaîne [...].

9. Beckwith, 1954.

10. Makaronas, 1963.

11. Les analyses ont été réalisées par Christophe Moulherat et Stella Spantidaki, et le rapport a été déposé au musée archéologique d'Arta.

12. Spantidaki, Moulherat, 2012, la pièce étudiée porte le n 3626 .

13. Alfaro Giner 1999, p. 137-138.

14. Inv. $n^{\circ} 350$, cette pièce a été étudiée par Youlie Spantidaki et Christophe Moulherat à la demande de Mme Stassinopoulou. Le rapport a été déposé au Musée national.
15. Wild, 1970.

16. Gleba, 2008, p. 70-75.

17. Pline, HN (VIII, 196).

18 Desrosiers, 1999, Bédat et al., 2005.

19. Moulherat C., Spantidaki S. 2012.

20. Moulherat, 2013, p. 303.

21. Pfister, 1934. Schmidt-Colinet, 2000.

\section{Références bibliographiques}

Alfro-Guiner C, 1999, «Archéologie des textiles anciens dans la péninsule Ibérique : à propos de quelques nouveautés» dans Cardon D. et Feugère M. (éds), Archéologie des textiles, des origines au $V^{e}$ siècle : actes du colloque de Lattes, octobre 1999. Montagnac, Monique Mergoil, 2000, Monographies instrumentum $\mathrm{n}^{\circ} 14,2000$, p. 131-140.

Bédat I., Desrosiers S., Moulherat C., Relier C., 2005, "Two Gallo-Roman Graves Recently Found in Naintré (Vienne, France)", in F. Pritchard and J.-P. Wild (eds), Northern Archaeological Textiles NESAT VII, p.5-11.

Caillou J. S., 2008, Les Tombeaux royaux de Judée dans l'Antiquité de David à Hérode Agrippa II. Essai d'archéologie funéraire, Geuthner, Paris.

Desrosiers S., 1999, « Textiles trouvés dans deux tombes du Bas-Empire à Naintré (Vienne) » dans Cardon D. et Feugère M. (éds), Archéologie des textiles, des origines au $V^{e}$ siècle : actes du colloque de Lattes, octobre 1999. Montagnac, Monique Mergoil, 2000, Monographies instrumentum $\mathrm{n}^{\circ} 14,2000$, p. 195-207.

Dussaud R., 1912, Les Monuments palestiniens et judaïques (Moab, Judée, Philistie, Samarie, Galilée), Ernest Leroux, Paris.

Gleba M., 2008, "Auratae vestes: Gold textiles in the ancient Mediterranean" dans Alfaro C. and Karali L. (eds), Purpureae Vestes II, Symposium Internacional sobre Textiles y Tintes del Mediterráneo en el mundo antiguo, p. 61-77, Valencia, University of Valencia.

Héron de Villefosse A., 1876, Notice des monuments provenant de la Palestine, Paris.
Makaronas C., 1963, « Tafoi para to Derbevni Qessa-lonivkh1 ", ArchDelt 18, p. 193-196.

Moulherat C., 2013, « Les vestiges textiles de la nécropole d'Egiin Gol », in Giscard P.-H., Turbat T. et Crubézy E. (dir.), Le premier Empire des steppes en Mongolie, éditions Faton, collection Archéomongolia, Paris, p. 297-312.

Moulherat C., Spantidaki S., 2012, « Les tissus à bandes d'or du Bas-Empire ; l'exemple de Thessaloniki " in Textiles and Dress in Greece and the Roman East : a Technological and Social Approach, p. 35-48. Kondyli Press, Kalamata.

Pfister R., 1934, Textiles de Palmyre, Les éditions d'art et d'Histoire, Paris.

Pline l'Ancien, Histoire naturelle, livre XXXIII, trad. Zehnacker, collection Budé, Les Belles Lettres, Paris, 1983.

Saulcy F. de, 1865, Voyage en Terre Sainte, Didier, Paris.

Saulcy F. de, 1867, Souvenirs d'un voyage en Terre Sainte, Librairie du Petit Journal, Paris.

Schmidt-Colinet A., Stauffer A., Al-Asad K., 2000, Die Textilien aus Palmyra. Neue und alte Funde. Ph. Von Zabern, Damaszener Forschungen 8, 201 p., 121 fig., Mayence.

Spantidaki Y., Moulherat C., 2012, "Greece", in Textiles and Textile Production in Europe: From Prehistory to AD 400 (Margarita Gleba and Ulla Mannering) (éds), Ancient Textiles Series 11, Oxbow Books in association with the Centre for Textile Research, 2012.

Wild J.-P., 1970, Textile manufacture in the northern Roman provinces, Cambridge, 1970 . 